\title{
Not Applicable Outcome
}

National Cancer Institute

\section{Source}

National Cancer Institute. Not Applicable Outcome. NCI Thesaurus. Code C159896.

The outcome is not applicable. 